Ann. Sci. forest., 1980, 37 (4), 373.

\title{
Observations récentes sur le complexe entomophage de Cryptococcus fagisuga
}

\author{
P. J. CHARLES * \\ Station d'Entomologie forestière, I.N.R.A., \\ Centre de Recherches d'Orléans \\ Olivet 45160 Ardon
}

De mai à septembre 1978, un étudiant du D.E.A. d'Entomologie de Paris-VI en stage à la Station de Zoologie Forestière d'Orléans, a effectué des observations sur la faune associée à Cryptococcus fagi dans les forêts de Lyons et d'Halatte, au moyen de brossages des troncs, piégeages et dénombrements à vue.

Aucun parasite entomophage n'a été trouvé.

Le complexe de prédateurs ne diffère pas sensiblement de ce qui est connu en Allemagne et en Grande-Bretagne.

Les principaux prédateurs sont les deux espèces de coccinelles, Exochomus quadripustulatus ( 99 p. 100 des spécimens observés) et Chilocorus renipustulatus.

Les autres prédateurs observés sont:

- un Diptère Cecidomyidae du genre Lestodiplosis qui fait l'objet de recherches plus poussées dans le travail poursuivi actuellement par $M$. Baylac au laboratoire d'Entomologie du Muséum National d'Histoire Naturelle à Paris, en relation avec le Pr Harris du British Museum,

- le Dermaptère Forficulidae Forficula auricularia, prédateur occasionnel,

- une espèce d'Hémiptère Anthocoridae. Themnosthetus gracilis et quelques chenilles d'un Lépidoptère Tineidae, dont le statut alimentaire (prédation ou saprophagie) n'est pas définitivement établi.

Les faibles effectifs de ces différents prédateurs qui ont pu être observés même sur des arbres portant de très fortes populations de cochenilles, nous inclinent à penser qu'ils n'ont qu'une très faible incidence sur la dynamique de populations de Cryptococcus dans les deux forêts étudiées.

* Intervention en séance : consulter BAYLAC M., 1980. Faune associée à Cryptococcus fagi (Baer.) (Homoptera : Coccoidea) dans quelques hêtraies du nord de la France. Acta oecologica, Oecol. applic., 1 (2): $199-208$. 